\title{
Approach to Improve Speed of Sound Calculation within PC-SAFT Framework
}

\author{
Liang, Xiaodong; Maribo-Mogensen, Bjørn; Thomsen, Kaj; Yan, Wei; Kontogeorgis, Georgios
}

Published in:

Industrial \& Engineering Chemistry Research

Link to article, DOI:

10.1021/ie3018127

Publication date:

2012

Document Version

Publisher's PDF, also known as Version of record

Link back to DTU Orbit

Citation (APA):

Liang, X., Maribo-Mogensen, B., Thomsen, K., Yan, W., \& Kontogeorgis, G. (2012). Approach to Improve Speed of Sound Calculation within PC-SAFT Framework. Industrial \& Engineering Chemistry Research, 51(45), 1490314914. https://doi.org/10.1021/ie3018127

\section{General rights}

Copyright and moral rights for the publications made accessible in the public portal are retained by the authors and/or other copyright owners and it is a condition of accessing publications that users recognise and abide by the legal requirements associated with these rights.

- Users may download and print one copy of any publication from the public portal for the purpose of private study or research.

- You may not further distribute the material or use it for any profit-making activity or commercial gain

- You may freely distribute the URL identifying the publication in the public portal 


\title{
Approach to Improve Speed of Sound Calculation within PC-SAFT Framework
}

\author{
Xiaodong Liang, ${ }^{\dagger}$ Bjørn Maribo-Mogensen, ${ }^{\dagger}$ Kaj Thomsen, ${ }^{\dagger}$ Wei Yan, ${ }^{\dagger}$ and Georgios M. Kontogeorgis ${ }^{*}{ }^{\dagger}$ \\ ${ }^{\dagger}$ Center for Energy Resources Engineering (CERE), Department of Chemical and Biochemical Engineering, Technical University of \\ Denmark, 2800 Kgs. Lyngby, Denmark \\ ${ }^{\ddagger}$ Center for Energy Resources Engineering (CERE), Department of Chemistry, Technical University of Denmark, 2800 Kgs. Lyngby, \\ Denmark
}

\section{Supporting Information}

ABSTRACT: An extensive comparison of SRK, CPA, and PC-SAFT for the speed of sound in normal alkanes has been performed. The results reveal that PC-SAFT captures the curvature of the speed of sound better than cubic EoS, but the accuracy is not satisfactory. Two approaches have been proposed to improve PC-SAFT's accuracy for speed of sound: (i) putting speed of sound data into parameter estimation; (ii) putting speed of sound data into both universal constants regression and parameter estimation. The results have shown that the second approach can significantly improve the speed of sound (3.2\%) prediction while keeping acceptable accuracy for the primary properties, i.e. vapor pressure $(2.1 \%)$ and liquid density (1.5\%). The two approaches have also been applied to methanol, and both give very good results.

\section{INTRODUCTION}

The speed of sound is used, in online measurements, to characterize the heterogeneous or homogeneous mixtures or to estimate the density of reservoir fluids down hole. ${ }^{1-5}$ Specifically SONAR (Sound Navigation and Ranging) uses sound propagation to navigate, communicate with, or detect objects on or under the surface of the water, and it can even provide some measurements of the echo characteristics of the "targets". ${ }^{6}$ An equation of state that describes the speed of sound for a wide range of mixtures accurately can be used to analyze these characteristics and then determine what they are. This will be very useful in quick detection of oil and gas leaks around subsea wells and in clear mapping of oil in seawater columns during the oil cleanup processing.

On the other hand, from the scientific point of view, as a second-order derivative property, the speed of sound is one of the most demanding tests to check the performance limits for a thermodynamic model, and from eq 1,7 it can be seen that its calculation needs accurate density or volume $(V)$, heat capacity, both isobaric $\left(C_{P}\right)$ and isochoric $\left(C_{V}\right)$, and the derivative of pressure with respect to total volume $(\partial P / \partial V)_{T, n}$.

$$
\begin{aligned}
& u=\sqrt{-\frac{V^{2}}{M_{\mathrm{w}}} \frac{C_{P}}{C_{V}}\left(\frac{\partial P}{\partial V}\right)_{T, n}} \\
& C_{V}=n\left(C_{P}^{\mathrm{ig}}-R\right)-T\left(\frac{\partial^{2} A^{\mathrm{r}}}{\partial T^{2}}\right)_{V, n} \\
& C_{P}=n\left(C_{P}^{\mathrm{ig}}-R\right)-T\left(\frac{\partial^{2} A^{\mathrm{r}}}{\partial T^{2}}\right)_{V, n}+T\left(\frac{\partial P}{\partial T}\right)_{V, n}\left(\frac{\partial V}{\partial T}\right)_{P, n}
\end{aligned}
$$

The details of how to calculate these derivatives analytically from an equation of state can be found in Chapter 2 (pages
63-65) of the book of Michelsen and Mollerup. ${ }^{7}$ In terms of Helmholtz free energy, the calculation of speed of sound needs its first- and second-order derivatives with respect to both temperature and total volume, and as pointed out by Gregorowicz et al., ${ }^{8}$ the precise description of the second derivative properties is a challenge for any EoS model. For instance, most of the classical equations of state (EoS), such as $\mathrm{SRK}^{9}$ and $\mathrm{PR}^{10}$ fail in describing speed of sound reliably in wide temperature and pressure range. $8,11,12$ This may be due to the intrinsic nature of these EoS, usually applied only to phase equilibria calculations, to the sensitivity of the second order derivative properties performed to a given function, or to the physics behind these properties. A way to discern some of these uncertainties could be to use a molecular-based EoS; these equations should ideally not depend on the particular properties under study and should retain the microscopic contributions considered when building the equation. Meanwhile it needs to be kept in mind that the second-order derivative properties should not be improved at the expense of significant deterioration of the primary properties, such as vapor pressure and liquid density.

The purposes of this work are (i) to review if the applications of SAFT models provide a "theoretically correct" approach to describe the first- and second-order derivative properties simultaneously based on literature; (ii) to validate whether the PC-SAFT model can capture the speed of sound curvature by comparing the results to $\mathrm{SRK}^{9}$ and $\mathrm{CPA}^{13-15}$ (iii) to study if it is possible to improve the performance of simplified PCSAFT $^{16-18}$ for speed of sound by putting this data into parameter estimation or by refitting the universal constants,

Received: July 9, 2012

Revised: September 28, 2012

Accepted: October 17, 2012

Published: October 18, 2012 
while keeping the same number of the pure component parameters.

Several applications of different SAFT approaches on speed of sound found in the literature are reviewed in section 2 . Following a short presentation of the models in section 3 , a preliminary comparison of existing models for normal hydrocarbons is presented in section 4. Two strategies are proposed and tested for improving PC-SAFT ${ }^{16-18}$ for speed of sound in section 5. After some preliminary results for methanol presented in section 6, a detailed discussion and conclusions are given in sections 7 and 8 . respectively.

\section{LITERATURE REVIEW ON THE APPLICATION OF SAFT MODELS ON THE SPEED OF SOUND}

Over the past two decades, the popularity of the statistical associating fluid theory (SAFT) EoS, based on a perturbation theory for associating fluids proposed by Wertheim, ${ }^{19-22}$ has grown very fast. The model appeared in the form known today due to the work of Chapman et al. ${ }^{23-25}$ and of Huang and Radosz $^{26,27}$ and for this reason both of these models are often referred to as "original SAFT". In the work of Huang and Radosz, the authors presented the parameters for many compounds and gave suggestion for the association schemes as well. After this, many different versions of SAFT have followed, some of the successful ones being the SAFT-VR in 1997 by Gil-Villegas et al., ${ }^{28,29}$ the soft-SAFT in 1997 by Blas and Vega, ${ }^{30-32}$ and the PC-SAFT in both its original version in 2001 by Gross and Sadowski ${ }^{16,17}$ and the simplified version in 2003 by von Solms et al. ${ }^{18}$

In SAFT, molecules are modeled as chains of covalently bonded spheres and the models are typically written as a sum of the contributions to the reduced residual Helmholtz free energy as in the form:

$$
a^{\mathrm{r}}=\frac{A^{\mathrm{r}}}{N k T}=a^{\text {seg }}+a^{\text {chain }}+a^{\text {assoc }}
$$

where $a^{\text {seg }}$ is the part of the Helmholtz energy due to segmentsegment interactions, $a^{\text {chain }}$ is the term due to chain formation, and $a^{\text {assoc }}$ represents the contribution due to association between different molecules, e.g. due to hydrogen bonding. The biggest differences in different SAFT variants are the dispersion term and the choice of reference fluid. Almost all of the different SAFT variants more or less use the same expressions for the chain formation and association terms and include in most cases five pure component parameters with well-defined physical meanings (the segment energy and size, the number of segments, and the association volume and energy). ${ }^{33}$

Nowadays this theoretical SAFT-type approach is very popular due to its versatility and the good results obtained for different applications. ${ }^{33}$ However, while SAFT's ability to describe the phase equilibria of chain and associating pure fluids and mixtures is well-established, its performance for the simultaneous estimation of first- and second-order derivative properties is still limited and not sufficiently explored. ${ }^{34}$ Some of the applications of SAFT family EoS for speed of sound calculations are reviewed below.

Lafitte et al. ${ }^{34,35}$ proposed the SAFT-VR Mie approach to simultaneously describe phase equilibria and derivative properties. In the first paper, they preliminarily checked the performance of the models PC-SAFT, ${ }^{16}$ SAFT-VR, ${ }^{28}$ and SAFT-VR LJC ${ }^{36}$ to describe the derivative properties with molecular parameters fitted to the vapor-liquid equilibrium data only, i.e. vapor pressure and saturated liquid densities, which aims to identify the limitations of these models. Poor agreement of the results from these models with the experimental data made them conclude that all these models fail to describe the speed of sound. As discussed in the article, it sounds like a feasible solution to recalculate molecular parameters for these models by taking into consideration isothermal compressibility data (or speed of sound) in the fitting procedure to overcome this problem. The reported results of these tests indicated a slight improvement on isothermal compressibility estimation results with an important deterioration of the vapor-liquid equilibrium curve. Hence, assuming that the problem in describing accurately the derivative properties was the choice of the intermolecular potential used to model the repulsion and dispersion interactions between the monomers forming the chain, they modified the potential term in the SAFT-VR approach, proposing the SAFT-VR Mie model, which introduces an extra compound-specific parameter related to the shape of the repulsive part of the potential. In addition, they proposed new fitting procedures to include two types of properties, vaporliquid equilibrium data, i.e. vapor pressure and liquid density, and the speed of sound in the condensed liquid phase. The results of both first- and second-order derivative properties were shown better agreements with the experimental data than the other SAFT models with original parameters. The mentioned \%AAD for the speed of sound was around $2 \%$. This work showed the capability of the SAFT-type models for describing both first- and second-derivative properties with good accuracy simultaneously. In the second article, ${ }^{35}$ they extended this approach to model vapor-liquid equilibria behavior and second-order derivative properties of alcohols and 1-alcohol $+n$-alkanes mixtures simultaneously. The extra nonconformal parameter characterizing the repulsive interaction between the monomer segments greatly enhanced the performance of the SAFT-VR theory for the prediction of second derivative properties of the 1-alcohol substances with around $2.5 \% \mathrm{AAD}$ for the speed of sound. This was due to the fact that in SAFT theory the contact radial distribution function of the segments plays a fundamental role not only in the chain contribution but also in the association contribution with the free energy. ${ }^{35}$

In order to get a more precise speed of sound prediction for mixtures from the SAFT-VR Mie model, Khammar and Shaw ${ }^{37}$ translated isentropic compressibility estimations for a mixture at a specific composition by adding the molar average error of the predicted pure components isentropic compressibility to the isentropic compressibility of the mixture predicted from SAFT-VR Mie EoS as follows:

$$
\begin{aligned}
\left.K_{s}\right|_{\text {ESTIM }}= & \left.\frac{1}{\rho_{\text {mix }} u_{\text {mix }}^{2}}\right|_{\text {SAFT }} \\
& +\sum_{i} x_{i}\left(\left.\frac{1}{\rho_{i} u_{i}^{2}}\right|_{\exp }-\left.\frac{1}{\rho_{i} u_{i}^{2}}\right|_{\text {SAFT }}\right)
\end{aligned}
$$

And then the speed of sound estimation was obtained from:

$$
\left.u\right|_{\text {ESTIM }}=\frac{1}{\sqrt{\left.\left.\rho_{\text {mix }}\right|_{\text {SAFT }} K_{s}\right|_{\text {ESTIM }}}}
$$


Llovell et al. ${ }^{38,39}$ argued that inclusion of properties other than vapor pressure and saturated liquid density data in the fitting procedure would reduce the predictive capability of the model. So they performed calculations with soft-SAFT ${ }^{30-32}$ on second derivative properties with the pure component parameters fitted to vapor pressure and saturated liquid density data only to show the physical soundness of the theory and to address specifically the transferability of the parameters. In addition, the soft-SAFT is able to accurately capture the density singularities related to the critical region by using a crossover treatment which explicitly incorporates a renormalization group term with two extra parameters. ${ }^{32}$ Pure $n$-alkanes and 1-alkanols were modeled in their first article. ${ }^{38}$ Their work provided a clear insight into the capability of the SAFT theory to capture simultaneously the vapor-liquid and derivative properties of an associating fluid, but the \%AAD of speed of sound for $n$-hexane and $n$-heptane at $T_{\mathrm{r}}=1.1$ are around $20 \%$. In the later work, ${ }^{39}$ the \%AADs of speed of sound for $n$-heptane at $0.1 \mathrm{MPa}$ and $101.3 \mathrm{MPa}$ is about $6 \%$.

Diamantonis et al. ${ }^{40}$ evaluated the performance of SAFT and PC-SAFT on derivative properties in a wide range of conditions for six fluids that are of interest to the Carbon Capture and Sequestration (CCS) technology. They used a similar approach, as that proposed by Llovell et al., ${ }^{38}$ to predict the second-derivative properties using the pure component parameters fitted to VLE data only. The results revealed that both models performed well, especially away from the critical region. PC-SAFT was shown to be more accurate than SAFT for $\mathrm{CO}_{2}, \mathrm{H}_{2} \mathrm{~S}$, and $\mathrm{H}_{2} \mathrm{O}$, while two models give comparable accuracies for other components. The average \%AAD of the PC-SAFT model on the speed of sound for the six fluids is $2 \%$. These results are not consistent with the point of view of Lafitte, ${ }^{34}$ who said that PC-SAFT was not able to describe the speed of sound well, but the author argued that direct comparisons are difficult, since the fluids and conditions examined are not the same. We agree partly with this argument and also want to point out that all the six fluids studied are composed of small molecules.

Very recently, to address the numerical pitfalls, unphysical predictions, and wrong estimations of the pure components' critical properties of the SAFT approaches, Polishuk ${ }^{41}$ proposed a SAFT plus Cubic approach, where in SAFT the attractive term of cubic EoS is attached as follows:

$$
A^{\mathrm{res}}=A^{\mathrm{res}, \mathrm{SAFT}}-\frac{a}{V+c}
$$

where $a$ and $c$ are additional fluid parameters.

As pointed out by the author, unlike other SAFT variants, SAFT + Cubic relies on generalizing the regularities exhibited by experimental data rather than approximating the results of molecular simulations. The authors concluded several merits of this approach: (i) free of the well-known disadvantages characteristic for several SAFT approaches, such as the inability to correlate the critical and subcritical pure compound data simultaneously and generating artificial unrealistic phase equilibria; (ii) free of numerical pitfalls; (iii) the smaller number of the pure compound adjustable parameters due to solving the critical conditions to obtain three of the five parameters (for most pure alkanes, one more parameter could be estimated by a empirical expression) when critical properties are available; (iv) relatively modest numerical contribution. This approach demonstrated its superiority on speed of sound calculation compared to SAFT-VR Mie, PC-SAFT, and SBWR for the selected systems both on curvature and accuracy from the figures in their published articles; ${ }^{41-44}$ unfortunately, they presented very limited \%AAD data explicitly, and also there is very little information about the vapor pressure prediction accuracy. This SAFT + Cubic approach has five parameters for nonassociating compounds and seven for associating ones.

In order to provide a comprehensive understanding of the potentials and limitations of the advanced SAFT family EoS and their improvements over classical models, Villiers et al. ${ }^{45,46}$ have studied the performance of SRK, PR, CPA, SAFT, and PC-SAFT on derivative properties for different component families, i.e., nonpolar, polar nonassociating, and associating, in both the compressed liquid and near-critical regions. On the basis of the fact that the total Helmholtz free energy is expressed as summation of separate contributions and all of the derivative properties could be calculated explicitly from one or more Helmholtz free energy derivatives with respect to temperature or total volume, they analyzed the contributions of individual terms on the final derivative properties and single derivatives. They concluded that, in general, the performance of PC-SAFT is superior in correlating most of the second-order derivative properties of investigated alkanes. A major improvement of the SAFT and PC-SAFT over CPA is its ability to give a better description of the $\mathrm{d} P / \mathrm{d} V$ derivative. However, as pointed out by the authors, this improvement is still not sufficiently accurate and is the primary reason why the models are not able to correlate the speed of sound accurately. They further pointed out that a similar approach as SAFT-VR $\mathrm{Mie}^{34,35}$ seems to be necessary in order to accurately predict speed of sound, since the $\mathrm{d} P / \mathrm{d} V$ derivative is predominantly influenced by the hard-sphere term and its incorrect slope with respect to pressure is possibly caused by the chain term, both of which are largely influenced by the radial distribution function. The similar incorrect slope of residual isochoric heat capacity and isothermal compressibility with respect to molar density from the chain term contribution were shown in the work of Llovell and Vega, ${ }^{38}$ in which they conducted the same term contribution analysis for these two properties for short and long, nonassociating and associating chain compounds. They concluded from this analysis that association played a dominate role in heat capacities (and other energetic properties) for relatively short associating chains.

On the basis of the literature investigations above, the SAFTtype models (the SAFT framework) seem to provide a "theoretically correct" approach to describe the first- and second-derivative properties simultaneously. The differences depend mostly on segment potentials, parameter estimation procedures, and the number of adjustable parameters used.

\section{MODELS}

In this section, the SRK, CPA, and SPC-SAFT EoS are used to evaluate the performance on the speed of sound. The CPA EoS, proposed by Kontogeorgis et al., ${ }^{13}$ is a model based on the SRK (or other cubic) EoS, widely used in the petroleum industry (e.g., for mixtures with gases and hydrocarbons), and on the SAFT theory for describing associating mixtures in a theoretically correct way. The CPA model reduces to SRK in the absence of hydrogen bonding compounds (water, alcohols, acids, etc.), thus achieving a balance between accuracy and simplicity and gaining acceptance in the oil, gas, and chemical industries. The main difference of these two models for nonassociating components comes from the parametrization. The critical properties and acentric factor are used in the SRK, 
while the pure component parameters of CPA are regressed from vapor pressure and liquid density. Besides simplicity and accuracy, the numerical implementation of the association term ensures that the computation time is not much higher than that of SRK and other simple models. $33,47,48$

The CPA EoS can be expressed for mixtures in terms of pressure $P$ as

$$
\begin{aligned}
P= & \frac{R T}{V_{\mathrm{m}}-b}-\frac{a(T)}{V_{\mathrm{m}}\left(V_{\mathrm{m}}+b\right)}-\frac{1}{2} \frac{R T}{V_{m}}\left(1+\rho \frac{\partial \ln g}{\partial \rho}\right) \\
& \sum_{i} x_{i} \sum_{A_{i}}\left(1-X_{A_{i}}\right)
\end{aligned}
$$

Where $\rho$ is the molar density $\left(\rho=1 / V_{\mathrm{m}}\right)$.

The details of SRK and CPA can be found in the book of Kontogeorgis and Folas. ${ }^{33}$

The sPC-SAFT model was proposed by von Solms et al. ${ }^{18}$ in order to simplify and reduce the computational time for the PC-SAFT EoS. In this respect, it is not a new EoS, rather a simplified version in terms of mixing rules of the original PCSAFT EoS, ${ }^{16}$ which means that the pure component parameters of the original and simplified PC-SAFT are the same. This work is mainly related to the dispersion term, and other terms can be found in the original literature or the book of Kontogeorgis and Folas. ${ }^{33}$

3.1. Dispersion Term. The Helmholtz energy for the dispersion term is given as the sum of a first-order and a second-order term:

$$
\begin{aligned}
& \frac{a^{\text {disp }}}{R T}=\frac{a_{1}^{\text {disp }}}{R T}+\frac{a_{2}^{\text {disp }}}{R T} \\
& \frac{a_{1}^{\text {disp }}}{R T}=-2 \pi \rho m^{2}\left(\frac{\varepsilon}{k T}\right) \sigma^{3} \int_{1}^{\infty} \tilde{u}(x) g^{\mathrm{hc}}(m ; x \sigma / d) x^{2} \mathrm{~d} x \\
& \frac{a_{2}^{\text {disp }}}{R T}=-\pi \rho m\left(1+Z^{\mathrm{hc}}+\rho \frac{\partial Z^{\mathrm{hc}}}{\partial \rho}\right)^{-1} m^{2}\left(\frac{\varepsilon}{k T}\right)^{2} \sigma^{3} \\
& \frac{\partial}{\partial \rho}\left[\rho \int_{1}^{\infty} \tilde{u}(x) g^{\mathrm{hc}}(m ; x \sigma / d) x^{2} \mathrm{~d} x\right] \\
& \left.1+Z^{\mathrm{hc}}+\rho \frac{\partial Z^{\mathrm{hc}}}{\partial \rho}\right) \\
& =\left(\begin{array}{l}
1+m \frac{8 \eta-2 \eta^{2}}{(1-\eta)^{4}}+(1-m) \\
\left.\frac{20 \eta-27 \eta^{2}+12 \eta^{3}-2 \eta^{4}}{[(1-\eta)(2-\eta)]^{2}}\right)
\end{array}\right.
\end{aligned}
$$

Where $x$ is the reduced radial distance around a segment $(x=$ $r / \sigma), \tilde{u}(x)=u(x) / \varepsilon$ denotes the reduced potential function, and $g^{\text {hc }}(m ; x \sigma / d)$ is the average segment-segment radial distribution function of the hard-chain fluid with temperaturedependent segment diameter $d(T)$.

Two important points are now the following: (i) the radial distribution function chains (rather than segment functions as before) and (ii) expressions for the two integrals, given below as power series in reduced density.

$$
\begin{aligned}
& I_{1}=\int_{1}^{\infty} \tilde{u}(x) g^{\mathrm{hc}}(m ; x \sigma / d) x^{2} \mathrm{~d} x=\sum_{i=0}^{6} a_{i} \eta^{i} \\
& I_{2}=\frac{\partial}{\partial \rho}\left[\rho \int_{1}^{\infty} \tilde{u}(x) g^{\mathrm{hc}}(m ; x \sigma / d) x^{2} \mathrm{~d} x\right]=\sum_{i=0}^{6} b_{i} \eta^{i}
\end{aligned}
$$

Where

$$
\begin{aligned}
& a_{i}=a_{0 i}+\frac{m-1}{m} a_{1 i}+\frac{m-1}{m} \frac{m-2}{m} a_{2 i} \\
& b_{i}=b_{0 i}+\frac{m-1}{m} b_{1 i}+\frac{m-1}{m} \frac{m-2}{m} b_{2 i}
\end{aligned}
$$

3.2. Average Absolute Deviation. In this work, the (percent) average absolute deviation is defined as

$$
\% \operatorname{AAD}(\Omega)=\frac{1}{N^{\exp }} \sum_{i=1}^{N^{\exp }}\left|\frac{\Omega^{\text {calc }}}{\Omega^{\exp }}-1\right| \times 100 \%
$$

where $\Omega$ is vapor pressure, liquid molar volume, or speed of sound.

\section{PRELIMINARY COMPARISON OF SRK, CPA, AND PC-SAFT}

Although some calculations of the SRK, CPA, and sPC-SAFT EoSs have been reported for the speed of sound for alkanes, ${ }^{46,49}$ it is still worth performing an extensive comparison for these models over wide temperature and pressure ranges. In this work, the performance of these three models on speed of sound is evaluated for the normal paraffins from methane to $n$ eicosane $\left(n-\mathrm{C}_{20}\right), n$-tetracosane $\left(n-\mathrm{C}_{24}\right)$, and $n$-hexatriacontane $\left(n-\mathrm{C}_{36}\right)$ over wide temperature and pressure ranges against the experimental or correlation data based on the available literature. The pure component parameters of CPA can be found in the book of Kontogeorgis and Folas, ${ }^{33}$ and those of PC-SAFT can be found in the original literature. ${ }^{16,59}$ The vapor pressure and saturated liquid density data is taken from the DIPPR correlations ${ }^{50}$ in the reduced temperature range $[0.45$, 0.9 ] for consistency and easy comparison with other models, while the speed of sound data of methane to $n$-decane is taken from the NIST database, ${ }^{51}$ and those of other long chain molecules are taken from the literature. ${ }^{52-58,60,61}$

The typical shapes of the constant temperature speed of sound curves from the three models are shown in Figures 1-3, from which it can be seen that sPC-SAFT performs better on capturing the curvature, although SRK or CPA have a better \% $\mathrm{AAD}$ in narrow low pressure ranges for some cases, such as hexane at $300 \mathrm{~K}$ in Figure 2. This is because the cancellation of the errors from the underpredicted to overpredicted regions. According to the investigation of Villiers, ${ }^{45,46}$ it is due to $\mathrm{SPC}$ SAFT providing a good description of the derivative of pressure with respect to volume, which is the dominant term in the speed of sound calculation. Not surprisingly, CPA performs significantly better to SRK as liquid density is used in parameter fitting procedure, which leads to a better description of speed of sound, as expressed in eq 1. It is shown in Figure 4, however, that the superiority of CPA over SRK is much smaller than that of sPC-SAFT over CPA, which indicates that the model itself is more important than the parameter fitting. The detailed \%AAD information of vapor pressure, liquid density, and speed of sound of these three models are supplied in Table S1 in the Supporting Information. 


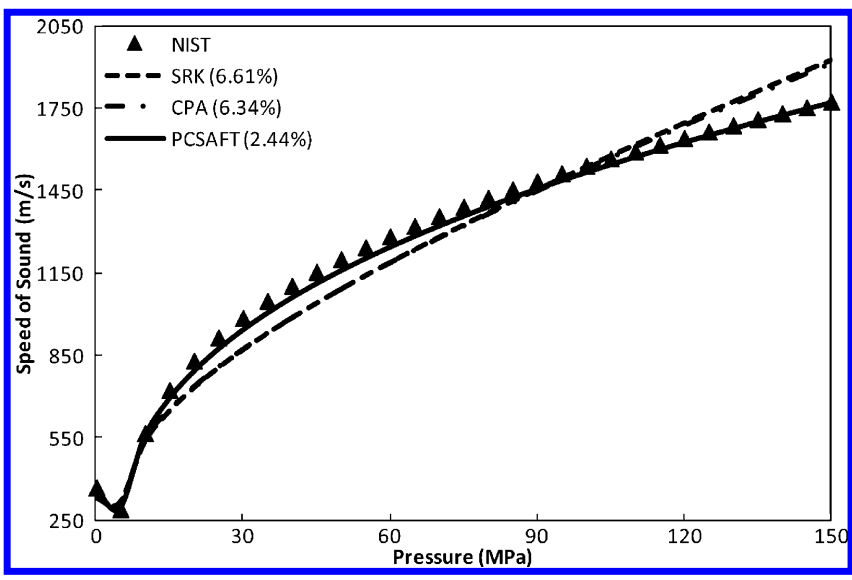

Figure 1. Speed of sound in methane at $T=200 \mathrm{~K}\left(T_{\mathrm{r}}=1.05\right)$. Triangles are data from NIST, ${ }^{51}$ and the dashed, dashed-dotted, and solid lines are results of SRK, CPA, and PC-SAFT, respectively.

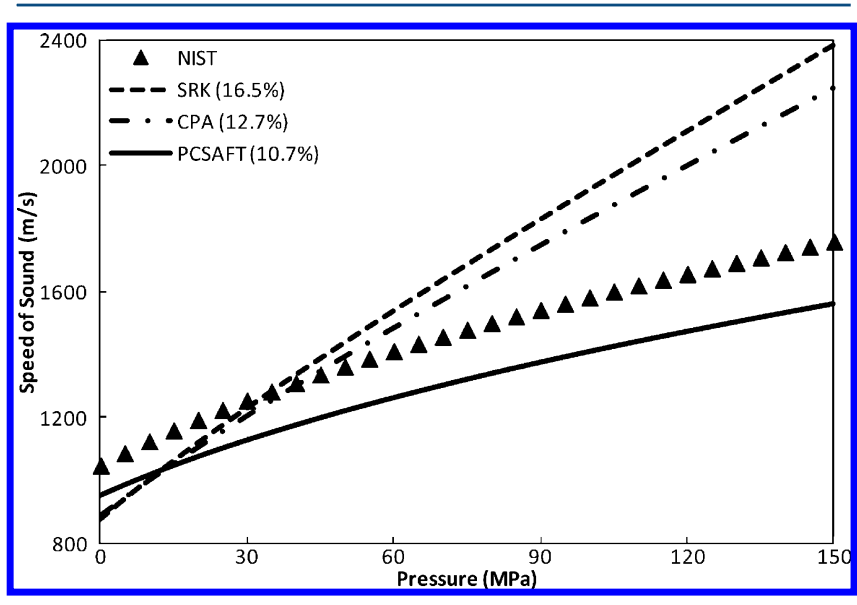

Figure 2. Speed of sound in $n$-hexane at $T=300 \mathrm{~K}\left(T_{\mathrm{r}}=0.59\right)$. Triangles are data from NIST, ${ }^{51}$ and the dashed, dashed-dotted, and solid lines are results of SRK, CPA, and PC-SAFT, respectively.

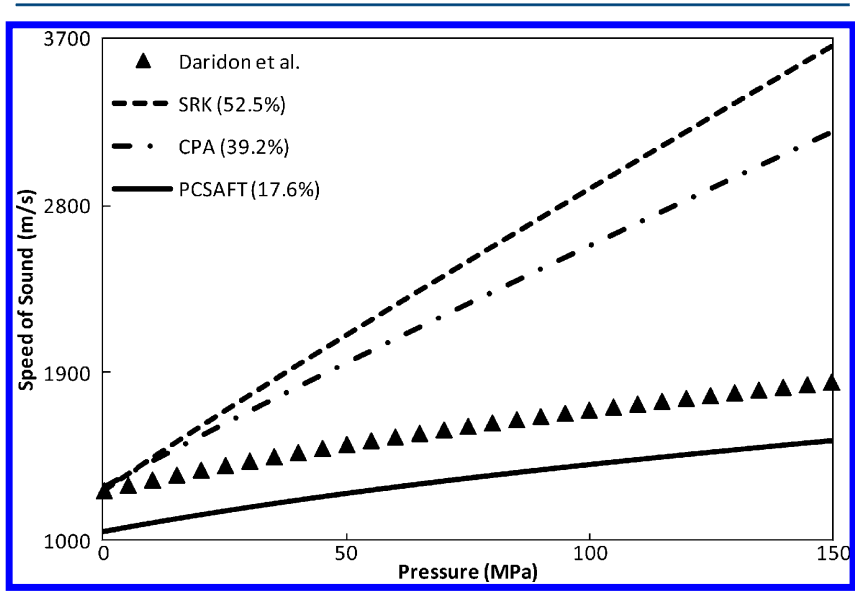

Figure 3. Speed of sound in $n$-pentadecane at $T=313.15 \mathrm{~K}\left(T_{\mathrm{r}}=\right.$ 0.44). Triangles are data from Daridon et al., ${ }^{55}$ and the dashed, dashed-dotted, and solid lines are results of SRK, CPA, and PCSAFT, respectively.

\section{APPROACHES TO IMPROVE THE PERFORMANCE OF PC-SAFT FOR THE SPEED OF SOUND}

PC-SAFT could be taken as a good start to calculate the speed of sound due to its capability of capturing the curvature as

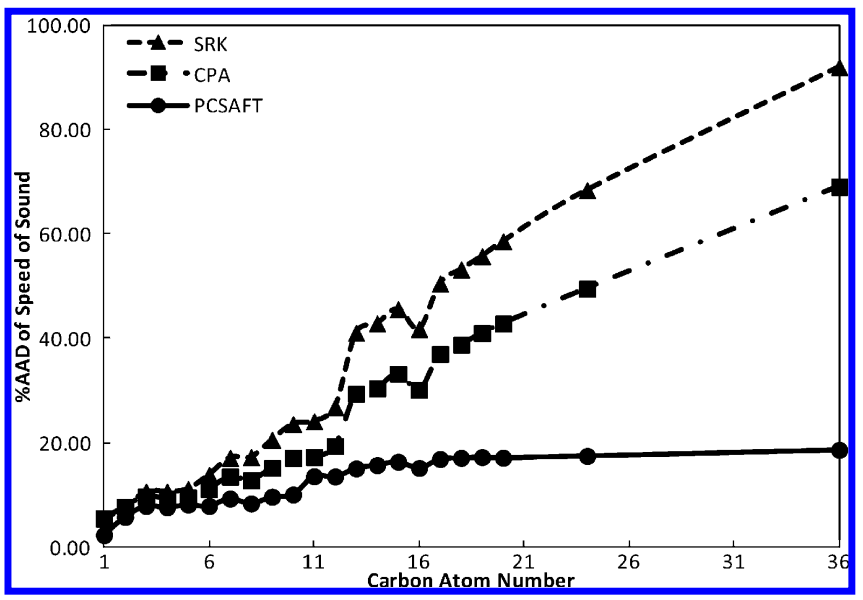

Figure 4. \%AAD between NIST data and model calculations of the speed of sound against carbon number. The dashed, dashed-dotted, and solid lines are results of SRK, CPA, and PC-SAFT, respectively.

discussed above. In this work, we have studied two approaches: (i) putting speed of sound data in the pure component parameters estimation; (ii) putting speed of sound data into both universal constants regression and pure component parameter estimation. They are denoted as approach 1 and 2 hereafter in this work.

5.1. Objective Function and Data. The objective function used in this work is

$$
\begin{aligned}
f_{\min }= & w_{p} \sum_{i}^{N_{p}}\left(\frac{P_{i}^{\exp }-P_{i}^{\text {calc }}}{P_{i}^{\exp }}\right)^{2}+w_{\rho} \sum_{i}^{N_{\rho}}\left(\frac{\rho_{i}^{\exp }-\rho_{i}^{\text {calc }}}{\rho_{i}^{\exp }}\right)^{2} \\
& +w_{u} \sum_{i}^{N_{u}}\left(\frac{u_{i}^{\exp }-u_{i}^{\text {calc }}}{u_{i}^{\exp }}\right)^{2}
\end{aligned}
$$

Where $P, \rho$, and $u$ are vapor pressure, liquid density, and speed of sound, respectively; $N$ and $w$ with the corresponding subscript are total experimental points and weights of the objective function, respectively. Equal weights are used in this preliminary study although Laffite ${ }^{34,35}$ suggested using half weight for the speed of sound in their parameter fitting procedure. This objective function is minimized by applying a Levenberg-Marquardt algorithm ${ }^{62}$ for both universal constant regression and pure component parameter estimation. Equal points of vapor pressure, liquid density, and speed of sound of saturated methane to $n$-decane are used for both universal constants regression and pure component parameters estimation. The points of vapor pressure and liquid density data used in the pure component parameter estimations depend on the available compressed liquid speed of sound data. The details of temperature and pressure ranges and how many data points can be found in Table 2 and Table $S 1$ in the Supporting Information.

5.2. Approach 1: Putting the Speed of Sound into Pure Component Parameters Estimation. To improve the performance of PC-SAFT for the speed of sound, an intuitively feasible approach is to take these data into consideration in the parameter fitting procedure, as mentioned by Laffite. ${ }^{34}$ The speed of sound data from saturated methane to $n$-decane $e^{51}$ and compressed $n$-undecane to $n$-hexatriacontane are used to estimate the pure component parameters, which along with the $\% \mathrm{AAD}$ of vapor pressure, liquid density, and speed of sound are listed in Tables 1 and 2 . There are two columns for the 
Table 1. PC-SAFT Pure Component Parameters Estimated from Different Approaches

\begin{tabular}{|c|c|c|c|c|c|c|c|c|c|}
\hline \multirow[b]{2}{*}{ alkanes } & \multicolumn{3}{|c|}{ original $^{16,59}$} & \multicolumn{3}{|c|}{ approach 1} & \multicolumn{3}{|c|}{ approach 2} \\
\hline & $m$ & $\sigma(\AA)$ & $\varepsilon / k(\mathrm{~K})$ & $m$ & $\sigma(\AA)$ & $\varepsilon / k(\mathrm{~K})$ & $m$ & $\sigma(\AA)$ & $\varepsilon / k(\mathrm{~K})$ \\
\hline $\mathrm{C} 1$ & 1.0 & 3.7039 & 150.03 & 1.0 & 3.7040 & 150.07 & 1.0 & 3.7030 & 149.98 \\
\hline $\mathrm{C} 2$ & 1.6069 & 3.5206 & 191.42 & 1.63742 & 3.4994 & 189.26 & 1.61691 & 3.5225 & 190.52 \\
\hline $\mathrm{C} 3$ & 2.002 & 3.6184 & 208.11 & 2.03288 & 3.5938 & 206.27 & 2.01124 & 3.6106 & 207.51 \\
\hline $\mathrm{C} 4$ & 2.3316 & 3.7086 & 222.88 & 2.41443 & 3.6633 & 218.20 & 2.3223 & 3.7129 & 223.40 \\
\hline $\mathrm{C} 5$ & 2.6896 & 3.7729 & 231.20 & 2.83126 & 3.6936 & 224.64 & 2.68579 & 3.7666 & 231.41 \\
\hline C6 & 3.0576 & 3.7983 & 236.77 & 3.29163 & 3.7014 & 227.18 & 3.0414 & 3.8083 & 237.15 \\
\hline C7 & 3.4831 & 3.8049 & 238.40 & 3.67278 & 3.7374 & 231.17 & 3.52812 & 3.7800 & 236.67 \\
\hline $\mathrm{C} 8$ & 3.8176 & 3.8373 & 242.78 & 4.23567 & 3.7096 & 229.48 & 3.89759 & 3.8137 & 239.76 \\
\hline C9 & 4.2079 & 3.8448 & 244.51 & 4.2586 & 3.8174 & 243.24 & 4.14894 & 3.8713 & 246.14 \\
\hline $\mathrm{C} 10$ & 4.6627 & 3.8384 & 243.87 & 4.90831 & 3.7797 & 237.26 & 4.62216 & 3.8560 & 244.73 \\
\hline $\mathrm{C} 11$ & 4.9082 & 3.8893 & 248.82 & 5.1315 & 3.8375 & 243.25 & 5.01574 & 3.8706 & 245.86 \\
\hline $\mathrm{C} 12$ & 5.3060 & 3.8959 & 249.21 & 5.78676 & 3.8548 & 245.09 & 5.3345 & 3.8907 & 247.88 \\
\hline $\mathrm{C} 13$ & 5.6877 & 3.9143 & 249.78 & 5.93966 & 3.8553 & 244.53 & 5.75866 & 3.8981 & 248.13 \\
\hline $\mathrm{C} 14$ & 5.9002 & 3.9396 & 254.21 & 6.26516 & 3.8737 & 246.61 & 6.06497 & 3.9194 & 250.39 \\
\hline $\mathrm{C} 15$ & 6.2855 & 3.9531 & 254.14 & 6.59888 & 3.8927 & 248.09 & 6.36243 & 3.9441 & 252.37 \\
\hline $\mathrm{C} 16$ & 6.6485 & 3.9552 & 254.70 & 6.95948 & 3.8986 & 249.12 & 6.74213 & 3.9457 & 252.77 \\
\hline $\mathrm{C} 17$ & 6.9809 & 3.9675 & 255.65 & 7.3613 & 3.9032 & 249.42 & 7.07933 & 3.9592 & 253.97 \\
\hline $\mathrm{C} 18$ & 7.3271 & 3.9668 & 256.20 & 7.73947 & 3.9077 & 249.59 & 7.43336 & 3.9661 & 254.25 \\
\hline C19 & 7.7175 & 3.9721 & 256.00 & 8.18528 & 3.8975 & 249.10 & 7.86483 & 3.9567 & 253.66 \\
\hline $\mathrm{C} 20$ & 7.9849 & 3.9869 & 257.75 & 8.53672 & 3.9155 & 249.90 & 8.19382 & 3.9759 & 254.60 \\
\hline $\mathrm{C} 24$ & 9.8220 & 3.9370 & 253.18 & 9.86769 & 3.9474 & 252.69 & 9.41287 & 4.0195 & 258.08 \\
\hline C36 & 13.86 & 4.0140 & 256.37 & 15.57073 & 3.8376 & 245.21 & 14.84568 & 3.9188 & 250.19 \\
\hline
\end{tabular}

Table 2. \%AAD with PC-SAFT of Vapor Pressure, Liquid Density, and Speed of Sound from Different Approaches ${ }^{a}$

\begin{tabular}{|c|c|c|c|c|c|c|c|c|c|c|c|c|c|c|c|}
\hline \multirow[b]{2}{*}{ subs } & \multicolumn{4}{|c|}{ original } & \multicolumn{4}{|c|}{ approach 1} & \multicolumn{4}{|c|}{ approach 2} & \multirow[b]{2}{*}{$T$ range $(\mathrm{K})$} & \multirow[b]{2}{*}{$\begin{array}{c}P \text { range } \\
(\mathrm{MPa})\end{array}$} & \multirow[b]{2}{*}{ ref } \\
\hline & $P$ & $\rho$ & $u(\mathrm{~s})$ & $u$ & $P$ & $\rho$ & $u(\mathrm{~s})$ & $u$ & $P$ & $\rho$ & $u(\mathrm{~s})$ & $u$ & & & \\
\hline $\mathrm{C} 1$ & 0.21 & 0.49 & 2.38 & 2.36 & 0.26 & 0.50 & 2.33 & 2.35 & 0.52 & 0.83 & 1.26 & 2.52 & $100-350$ & $0.1-150$ & $16,50,51$ \\
\hline $\mathrm{C} 2$ & 0.25 & 0.68 & 7.83 & 5.80 & 0.50 & 0.40 & 7.79 & 5.75 & 0.68 & 1.75 & 0.90 & 3.69 & $150-500$ & $0.1-150$ & $16,50,51$ \\
\hline $\mathrm{C} 3$ & 0.19 & 0.51 & 8.69 & 7.92 & 0.62 & 0.39 & 8.59 & 8.00 & 0.65 & 1.90 & 1.54 & 3.55 & $150-500$ & $0.1-150$ & $16,50,51$ \\
\hline $\mathrm{C} 4$ & 0.33 & 0.50 & 8.55 & 7.66 & 1.62 & 0.72 & 8.57 & 7.73 & 0.72 & 1.80 & 1.34 & 3.32 & $200-550$ & $0.1-150$ & $16,50,51$ \\
\hline $\mathrm{C} 5$ & 0.29 & 1.35 & 8.84 & 8.18 & 1.86 & 0.69 & 8.58 & 8.42 & 0.58 & 1.59 & 1.68 & 3.51 & $250-550$ & $0.1-150$ & $16,50,51$ \\
\hline C6 & 1.05 & 0.66 & 7.04 & 7.92 & 2.74 & 1.25 & 6.79 & 7.99 & 1.74 & 1.49 & 0.58 & 2.97 & $250-600$ & $0.1-150$ & $16,50,51$ \\
\hline $\mathrm{C} 7$ & 0.26 & 0.94 & 8.40 & 9.34 & 2.61 & 1.73 & 8.44 & 9.42 & 1.13 & 1.63 & 1.52 & 3.47 & $250-600$ & $0.1-150$ & $16,50,51$ \\
\hline $\mathrm{C} 8$ & 0.44 & 0.82 & 7.33 & 8.40 & 4.32 & 2.48 & 6.78 & 8.34 & 1.16 & 1.56 & 0.41 & 2.69 & $300-600$ & $0.1-150$ & $16,50,51$ \\
\hline $\mathrm{C} 9$ & 0.77 & 0.62 & 8.73 & 9.69 & 0.99 & 0.61 & 8.56 & 9.82 & 1.40 & 1.82 & 2.30 & 2.91 & $300-600$ & $0.1-150$ & $16,50,51$ \\
\hline $\mathrm{C} 10$ & 0.84 & 0.61 & 8.42 & 10.1 & 3.02 & 1.91 & 8.23 & 10.1 & 1.28 & 1.59 & 1.54 & 3.93 & $300-650$ & $0.1-150$ & $16,50,51$ \\
\hline $\mathrm{C} 11$ & 1.79 & 0.67 & & 13.6 & 1.32 & 1.44 & & 13.3 & 1.52 & 1.41 & & 2.03 & $303.15-413.15$ & $0.1-118$ & $16,50,52$ \\
\hline $\mathrm{C} 12$ & 1.29 & 0.79 & & 13.6 & 1.25 & 1.40 & & 13.3 & 1.52 & 1.41 & & 2.07 & $293.15-433.15$ & $0.1-140$ & $16,50,53$ \\
\hline $\mathrm{C} 13$ & 1.89 & 1.18 & & 15.1 & 1.29 & 1.70 & & 14.8 & 1.37 & 1.37 & & 2.24 & $293.15-433.15$ & $0.1-150$ & $16,50,54$ \\
\hline $\mathrm{C} 14$ & 2.69 & 0.74 & & 15.7 & 1.43 & 1.70 & & 15.1 & 1.43 & 1.37 & & 2.52 & $293.15-433.15$ & $0.1-150$ & $16,50,54$ \\
\hline $\mathrm{C} 15$ & 2.24 & 0.79 & & 16.4 & 1.13 & 1.78 & & 16.0 & 1.11 & 1.37 & & 2.94 & $293.15-383.15$ & $0.1-150$ & $16,50,55$ \\
\hline $\mathrm{C} 16$ & 2.51 & 0.63 & & 15.2 & 1.39 & 1.54 & & 14.8 & 1.77 & 1.33 & & 2.79 & $303.15-433.15$ & $0.1-140$ & $16,50,56$ \\
\hline $\mathrm{C} 17$ & 2.95 & 0.58 & & 16.9 & 1.26 & 1.80 & & 16.4 & 1.54 & 1.29 & & 3.26 & $303.15-383.15$ & $0.1-150$ & $16,50,55$ \\
\hline $\mathrm{C} 18$ & 3.01 & 0.51 & & 17.1 & 1.20 & 1.82 & & 16.5 & 1.49 & 1.28 & & 3.47 & $313.15-383.15$ & $0.1-150$ & $16,50,57$ \\
\hline C19 & 3.71 & 0.51 & & 17.3 & 1.70 & 1.73 & & 16.7 & 2.21 & 1.26 & & 3.66 & $313.15-383.15$ & $0.1-150$ & $16,50,57$ \\
\hline $\mathrm{C} 20$ & 4.17 & 0.79 & & 17.2 & 1.86 & 1.82 & & 16.4 & 2.71 & 1.24 & & 3.46 & $323.15-393.15$ & $0.1-150$ & $16,50,58$ \\
\hline $\mathrm{C} 24$ & 1.28 & 0.41 & & 17.5 & 1.09 & 1.76 & & 17.3 & 2.57 & 1.25 & & 4.25 & $333.15-393.15$ & $0.1-150$ & $50,59,60$ \\
\hline $\mathrm{C} 36$ & 19.4 & 1.49 & & 18.7 & 16.5 & 2.23 & & 17.5 & 17.2 & 1.91 & & 5.10 & $363.15-403.15$ & $0.1-151$ & $50,59,61$ \\
\hline $\operatorname{Avg}^{1}$ & 0.46 & 0.72 & 7.62 & 7.74 & 1.85 & 1.07 & 7.47 & 7.79 & 0.99 & 1.60 & 1.31 & 3.26 & & & \\
\hline $\mathrm{Avg}^{2}$ & 2.34 & 0.74 & & 12.4 & 2.27 & 1.43 & & 12.1 & 2.10 & 1.48 & & 3.20 & & & \\
\hline
\end{tabular}

$a_{\text {The }} u(\mathrm{~s})$ denotes the \%AAD value only for saturated data of methane to $n$-decane. Refs 16 and 59 refer to the pure component parameters of the original PC-SAFT. Avg ${ }^{1}$ means average deviation from methane to $n$-decane. $\mathrm{Avg}^{2}$ means average deviation from methane to $n$-hexatriacontane.

speed of sound in Table 2 , and the one with $u$ (s) means that the \%AAD is calculated for saturated speed of sound data, since this data is used to fit the parameters for methane to $n$-decane. The two avg rows in Table 2 are for methane to $n$-decane and for methane to $n$-hexatriacontane, respectively, since the data of methane to $n$-decane are used to readjust the universal constants. The large \%AAD vapor pressure of $n$-hexatriacontane is mainly because its vapor pressure from DIPPR 
correlation is about $4.0 \times 10^{-4} \mathrm{~Pa}$ at $T_{\mathrm{r}}=0.45$. Similar results were reported for SAFT-VR and SAFT-VR Mie. ${ }^{34}$

5.3. Approach 2: Putting the Speed of Sound into Both Universal Constant Regression and Pure Component Parameters Estimation. As shown in Figures 5 and 6,

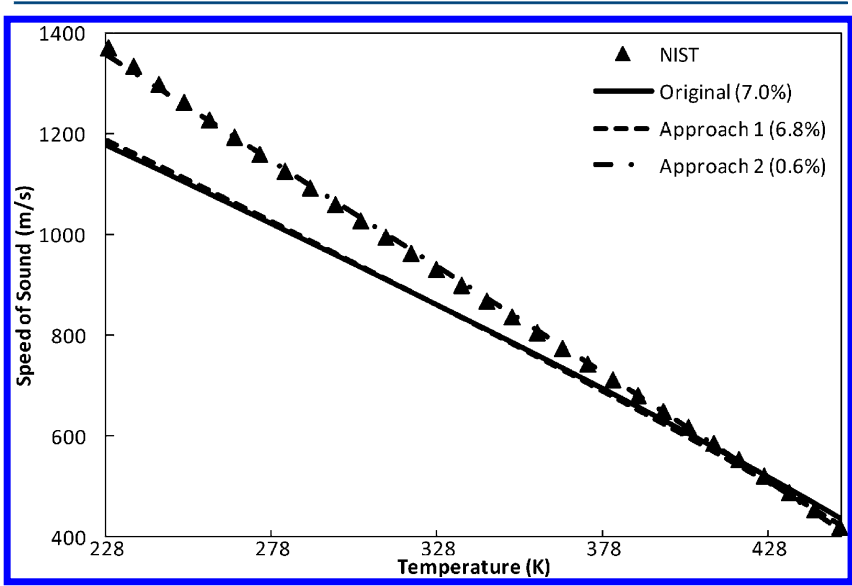

Figure 5. Speed of sound in saturated liquid $n$-hexane with PC-SAFT from different approaches. Triangles are data from NIST. ${ }^{51}$ The solid, dashed, and dashed-dotted line are results of the original model, approach 1 , and approach 2 , respectively.

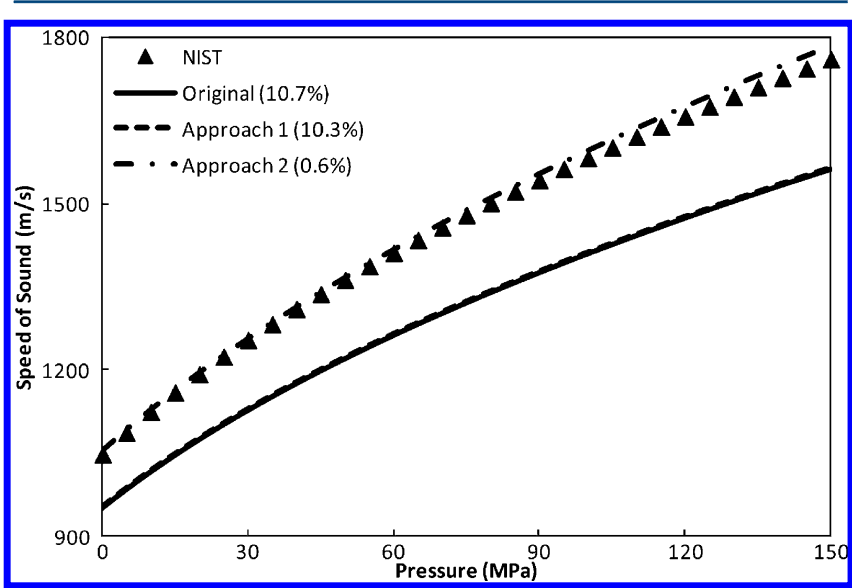

Figure 6. Speed of sound in $n$-hexane at $300 \mathrm{~K}$ with PC-SAFT from different approaches. Triangles are data from NIST. ${ }^{51}$ The solid, dashed, and dashed-dotted lines are results of original model, approach 1 , and approach 2 , respectively.

the speed of sound calculated from the original sPC-SAFT model does not deviate qualitatively very much from the experimental data curve. Thus, it is speculated whether it is possible to "rotate" or "move" somewhat the calculated curve in order to match the experimental results better by putting the speed of sound data into the universal constants regression.

It can be seen from eqs 15 and 16 that only 14, i.e. $\left\{a_{0 i}\right\}$ and $\left\{b_{0 i}\right\}$, of the 42 universal constants need to be fitted for methane if its segment number is fixed to 1 . On the other hand, as shown in Figure 1, sPC-SAFT can predict the speed of sound for methane with good accuracy. So we propose to fit the universal constants and pure component parameters in two steps. In the first step, the universal constants and pure component parameters are regressed for methane using an iterative procedure as shown below: (i) Estimate the pure component parameters with old universal constants

(ii) Regress the coefficients $\left\{a_{i}\right\},\left\{b_{i}\right\}$ for each component

(iii) Estimate the pure component parameters with the new universal constants

(iv) Repeat steps ii to iii until convergence is obtained

The original $\left\{a_{0 i}\right\}$ and $\left\{b_{0 i}\right\}$ provide good initial estimates for the regression. In the second step, only the differences of the coefficients from those of methane need to be regressed, i.e., the sum of the last two terms of eqs 15 and 16 . The same procedure is applied for ethane to $n$-decane, but an additional step is needed to fit the coefficients to segment number $m$ after getting the individual coefficients, in which step 28 , i.e. $\left\{a_{1 i}, a_{2 i}\right\}$ and $\left\{b_{1 i}, b_{2 i}\right\}$ in eqs 15 and 16 , of the 42 universal constants can be fitted. This procedure makes it possible to use the original universal constants as good initial values safely. The same data, saturated liquid speed of sound data of methane to $n$-decane, and the same objective function (18) are used for both universal constants regression and pure component parameters estimation, which are solved by a Levenberg-Marquardt algorithm. $^{62}$

Convergence here means that the changes of overall \%AAD of the three properties or the changes of the pure component parameters are very small, for instance less than $1.0 \times 10^{-3}$. It is unavoidable to arrive to multiple local minimum points when the problem has several parameters, as discussed later in the Discussion section. Thus it is a good strategy to decrease the tolerance in the convergence criteria error gradually and to keep the curves on reasonable trends which can be controlled by carefully choosing boundaries for the coefficients.

The new universal constants can be found in Table S2 in the Supporting Information, and the pure component parameters and $\% \mathrm{AAD}$ of the three concerned properties $\left(P^{\text {sat }}, \rho, u\right)$ are also shown in Tables 1 and 2 along with the other two approaches. The speed of sound of saturated and compressed $n$-hexane and compressed $n$-pentadecane are shown in Figures 5-7 with the three approaches.

\section{PRELIMINARY RESULTS OF METHANOL}

It is worth investigating the impact of the new universal constants on associating compounds such as methanol. The same three approaches are used for methanol using the $2 \mathrm{~B}$ association scheme and the \%AAD of vapor pressure, liquid density, and speed of sound are reported in Table 3 . The same temperature range and source data as for SAFT-VR Mie model $^{35}$ are used in order to compare the PC-SAFT and SAFTVR Mie.

It is seen from the results in Table 3 that approach 1 can give very good \%AAD for all three properties, which was also observed by Villiers. ${ }^{46}$ These results are comparable to those calculated from the SAFT-VR Mie model ${ }^{35}$ with one pure component parameter less. Meanwhile, Approach 2 could indeed capture the curvature of speed of sound a bit better as shown in Figure 8.

\section{DISCUSSION}

Approach 1 offers small improvements on the speed of sound but yields poor vapor pressures and liquid densities, especially for methane to $n$-decane, as shown in Table 2 . Similar results were obtained in the works of Villiers ${ }^{45,46}$ and Laffite. ${ }^{34}$ With approach 2, significant improvements are obtained both in terms of accuracy and reproducing the curvature of the speed of 


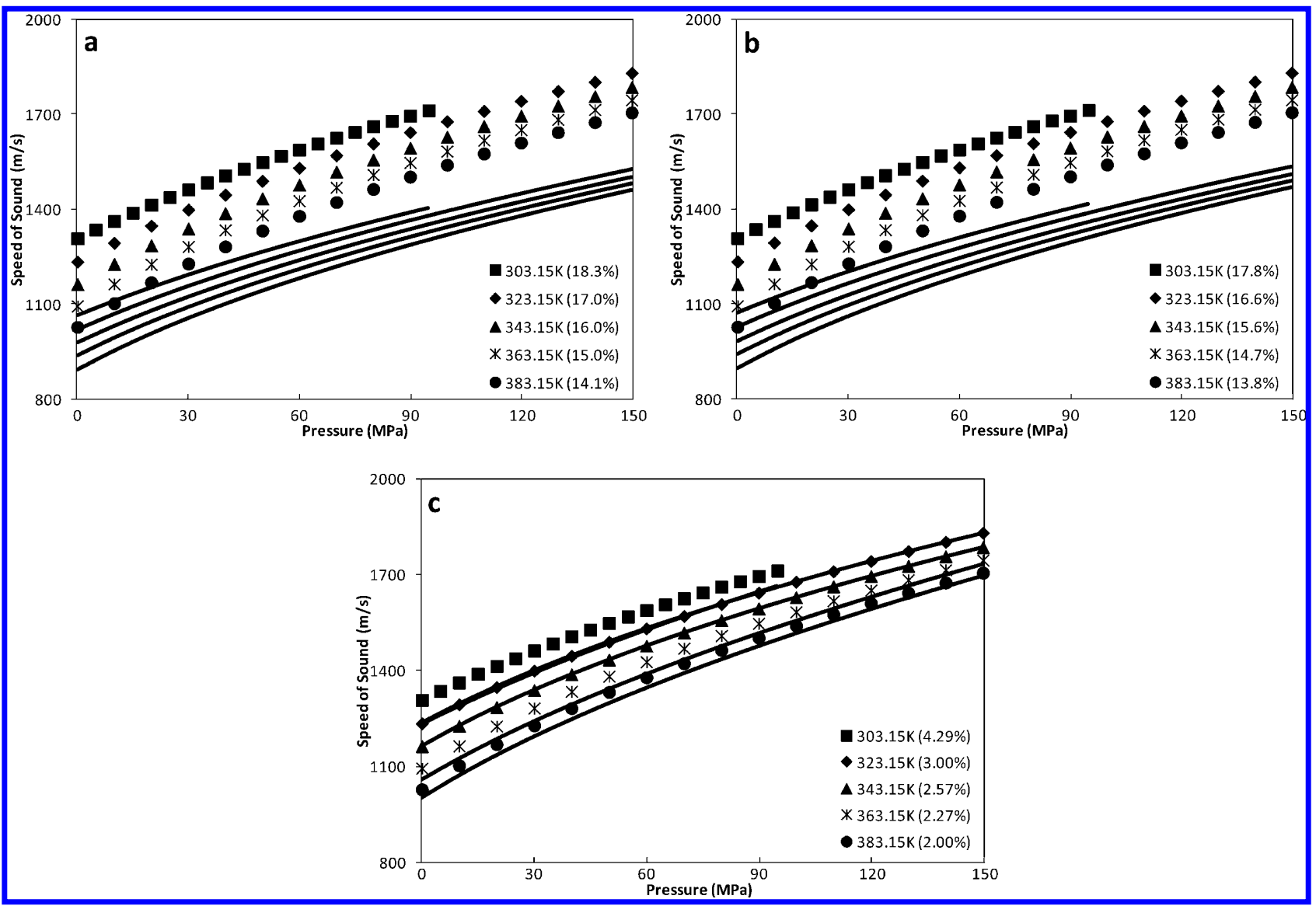

Figure 7. Speed of sound in $n$-pentadecane with PC-SAFT. Comparisons of experimental data from Daridon et al. ${ }^{55}$ (points) with calculation results (curves) of the original model (a), approach 1 (b), and approach 2 (c). The points and curves correspond to the temperature from top to bottom.

Table 3. Parameters and \%AAD of Properties of Methanol from Different PC-SAFT Approaches

\begin{tabular}{|c|c|c|c|c|c|c|c|c|c|}
\hline \multirow[b]{2}{*}{ method } & \multicolumn{5}{|c|}{ parameters } & \multicolumn{3}{|c|}{$\% \mathrm{AAD}$} & \multirow[b]{2}{*}{ ref } \\
\hline & $m$ & $\sigma(\AA)$ & $\varepsilon / k(\mathrm{~K})$ & $\varepsilon / k^{A i B j}(\mathrm{~K})$ & $\kappa^{A i B j}$ & $P^{\text {sat }}$ & $\rho^{\text {sat }}$ & $u$ & \\
\hline original & 1.5255 & 3.23 & 188.9 & 2899.5 & 0.035176 & 1.86 & 0.53 & 5.52 & 17,63 \\
\hline approach 1 & 1.88238 & 3.0023 & 181.77 & 2738.03 & 0.054664 & 1.39 & 0.22 & 1.19 & 63 \\
\hline approach 2 & 1.55166 & 3.2335 & 190.66 & 2864.78 & 0.035924 & 1.54 & 0.58 & 0.73 & 63 \\
\hline$T$ range $(\mathrm{K})$ & \multirow{2}{*}{\multicolumn{5}{|c|}{ 256-461 (saturation temperature range) }} & \multicolumn{3}{|c|}{$303.15-373.15(u)$} & \\
\hline$P$ range $(\mathrm{MPa})$ & & & & & & $0.1-$ & & & \\
\hline
\end{tabular}

sound, as seen in Table 2 and Figures 5-7, with a small loss in accuracy for vapor pressures and liquid densities. Compared to the SAFT-VR Mie model, ${ }^{34}$ our approach is slightly inferior on both saturated liquid density and speed of sound based on the limited data from their work but is better on vapor pressures. The average \%AAD of vapor pressure and liquid density of the same $n$-alkanes from SAFT-VR Mie model are $5.0 \%$ and $0.6 \%$, respectively, while the reported average \%AAD of speed of sound was close to $2 \%$. (The condensed liquid density data was used in parameter estimation for SAFT-VR Mie). Meanwhile our model captures the speed of sound curvature a bit better, especially in high pressure ranges, comparing to the corresponding figures for $n$-pentadecane in the SAFT-VR Mie work. $^{34}$ There are only three pure component parameters for nonassociating components in our approach.

Due to their clear physical meanings and the same PC-SAFT framework, it can be seen from Table 1 that the three pure component parameters obtained from all three approaches are close to each other and follow similar trends with molecular weight. As shown in Figure 9, the parameters $m, m \sigma^{3}$, and $m \varepsilon / k$ of approach 2 are all linear functions of molecular weight, which allows the prediction of parameters for heavier molecular weight compounds from knowledge of similar shorter chain compounds. $^{33}$ The detailed linear correlations of these parameters from all three approaches can be found in Table S3 in the Supporting Information.

For short associating compounds, e.g. methanol, as shown in the work of Llovell and Vega, ${ }^{38}$ the association plays an important role in derivative properties. From the parameter estimation point of view, two additional parameters give significant flexibility to fit the experimental data. As shown in Table 3, approach 1 yields better overall \%AAD than those of both original and approach 2 models. We can see that the parameters with approach 1 differ more than those of approach 2 compared to the original ones. As argued by Lafitte, ${ }^{35}$ any theory with a certain degree of complexity including several characteristic parameters must face the presence of several local minima for the fitted parameters. The resulting values after any 


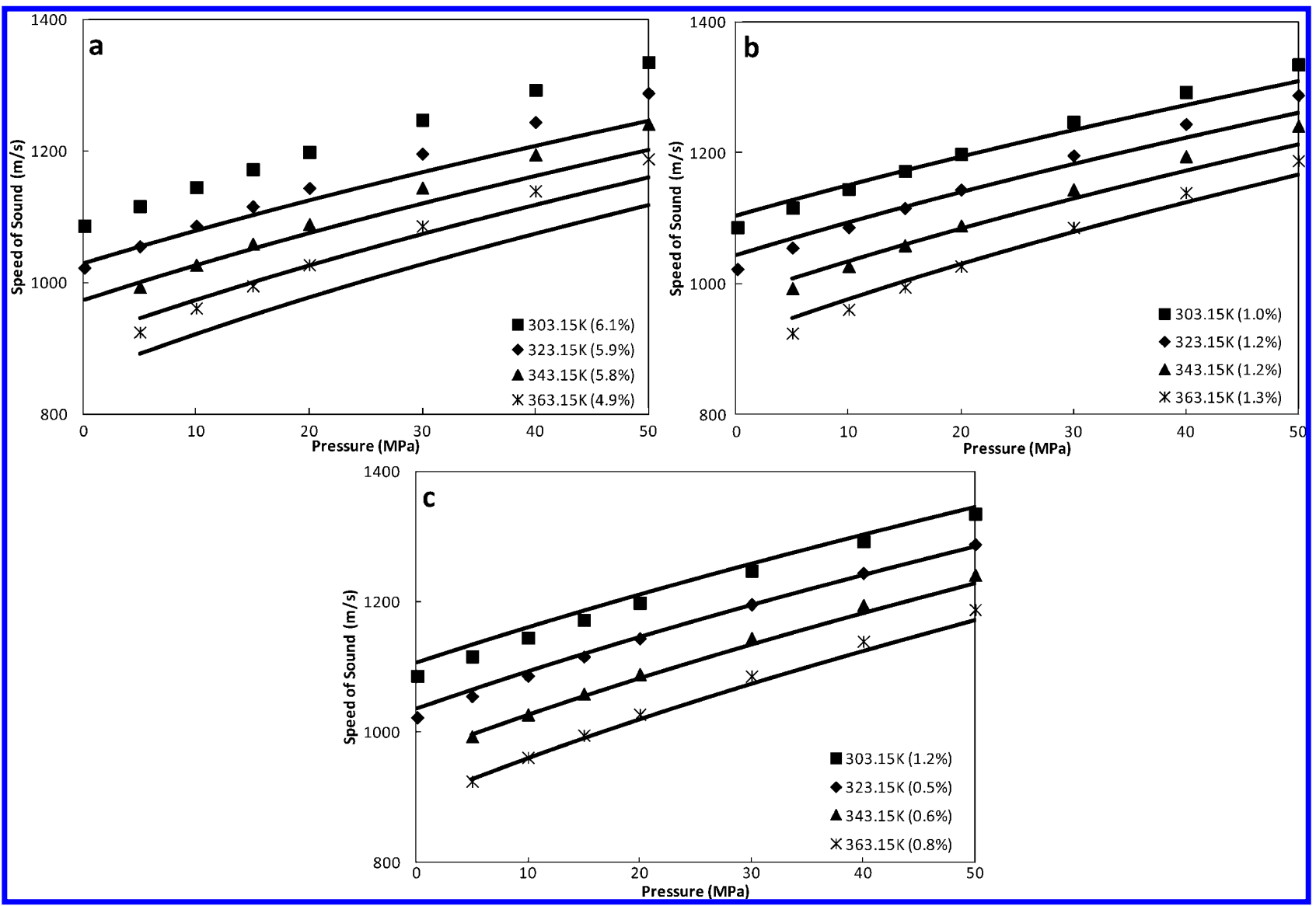

Figure 8. Speed of sound in methanol with PC-SAFT. Comparison of experimental data ${ }^{63}$ (points) with calculation results (curves) of the original model (a), approach 1 (b), and approach 2 (c).

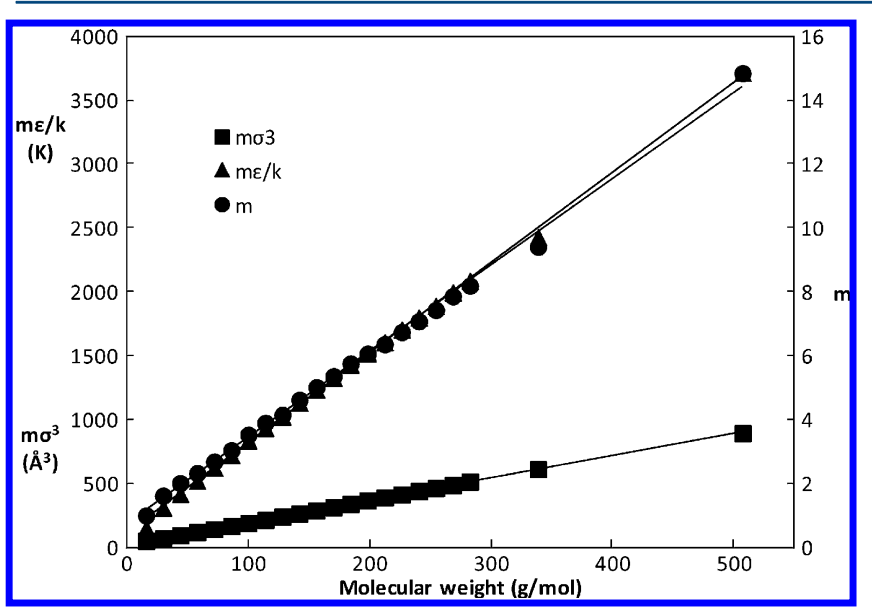

Figure 9. Parameter groups $m$ (cycle), $m \sigma^{3}$ (square), and $m \varepsilon / k$ (triangle) of approach 2 as linear functions of molecular weight for $n$ alkanes up to $n-C_{36}$. Lines are linear fits to these points.

correlation depend to a great extent on a priori conditions imposed on the parameters, as their physical meanings and the choice of the objective functions. Hence, the values of the parameters will depend strongly on what we want to estimate and to the expected degree of accuracy. Similar results were reported by Avlund. ${ }^{64}$ This fact reveals that it is a good strategy to find a better solution with more inherently different constraints, for instance by putting second derivative properties into the parameter fitting procedure, when there are extra parameters, such as association energy and volume here, and interaction width in the SAFT-VR model. However, for considerable long associating chain molecules, i.e. longer than 1-hexanol, Approach 1 offers a small improvement on speed of sound with lager deviations on primary properties (brief results are supplied in Table S4 in the Supporting Information). This is because the dominant contribution is turned to be the chain length for long chain molecules, which is consistent with the results of Llovell and Vega. ${ }^{38}$

To test the overall performance of approach 2, we calculated the molar volumes, isochoric and isobaric heat capacity, speed of sound, and the derivative of pressure with respect to volume in the same temperature and pressure ranges of speed of sound in Table 2 for methane to $n$-decane. These properties are directly involved in eqs $1-3$, and these calculations are predictive. As shown in Table 4, approach 2 improves the speed of sound and the derivative of pressure with respect to volume while deteriorating the molar volume and isobaric heat capacity. Both models give comparable isochoric heat capacity, which reveals that the new approach does have impact on the derivatives of Helmholtz free energy with respect to volume, but less so on temperature related derivatives from eqs $1-3{ }^{7}$ It is interesting to see that the original PC-SAFT describes the isobaric heat capacity for hydrocarbons very well, but it can be shown that that the new approach gives comparable isobaric heat capacity results as SAFT-VR Mie ${ }^{34}$ (See the Supporting Information for detailed isobaric heat capacity calculation results of these three models ${ }^{65-67}$ ). In the next step, it is worth investigating how the performance of the models on the derivatives of Helmholtz free energy with respect to temper- 
Table 4. \%AAD of Different Properties with Original PC-SAFT and Approach 2 over Wide Temperature and Pressure Ranges

\begin{tabular}{|c|c|c|c|c|c|c|c|c|c|c|}
\hline \multirow[b]{2}{*}{ alkanes } & \multicolumn{5}{|c|}{ original PC-SAFT } & \multicolumn{5}{|c|}{ PC-SAFT, approach 2} \\
\hline & $\rho$ & $u$ & $C_{\mathrm{V}}$ & $C_{\mathrm{P}}$ & $\mathrm{d} P / \mathrm{d} V$ & $\rho$ & $u$ & $C_{\mathrm{V}}$ & $C_{\mathrm{P}}$ & $\mathrm{d} P / \mathrm{d} V$ \\
\hline $\mathrm{C} 1$ & 0.99 & 2.36 & 3.42 & 1.36 & 3.62 & 1.2 & 2.52 & 3.40 & 1.99 & 5.82 \\
\hline $\mathrm{C} 2$ & 1.74 & 5.80 & 4.77 & 1.23 & 4.76 & 1.85 & 3.69 & 5.05 & 3.65 & 11.15 \\
\hline C3 & 1.44 & 7.95 & 5.24 & 1.43 & 9.32 & 2.33 & 3.55 & 5.54 & 4.28 & 10.9 \\
\hline $\mathrm{C} 4$ & 1.40 & 7.66 & 4.91 & 1.44 & 9.47 & 2.23 & 3.32 & 4.76 & 3.37 & 9.72 \\
\hline $\mathrm{C} 5$ & 1.11 & 8.18 & 4.38 & 1.06 & 11.1 & 2.1 & 3.51 & 4.15 & 3.17 & 8.27 \\
\hline C6 & 1.35 & 7.92 & 3.82 & 1.25 & 10.4 & 2.14 & 2.99 & 3.48 & 3.14 & 8.82 \\
\hline $\mathrm{C} 7$ & 1.29 & 9.34 & 3.22 & 1.06 & 13.6 & 2.21 & 3.47 & 2.86 & 3.74 & 7.48 \\
\hline $\mathrm{C} 8$ & 1.31 & 8.40 & 1.99 & 0.82 & 12.0 & 1.69 & 2.69 & 1.70 & 3.43 & 6.59 \\
\hline C9 & 1.73 & 9.69 & 2.73 & 0.89 & 13.0 & 2.02 & 2.91 & 2.26 & 3.86 & 5.80 \\
\hline $\mathrm{C} 10$ & 1.63 & 10.12 & 2.60 & 0.96 & 14.5 & 2.1 & 3.93 & 2.13 & 3.62 & 5.39 \\
\hline avg. & 1.40 & 7.74 & 3.71 & 1.15 & 10.2 & 1.99 & 3.26 & 3.53 & 3.43 & 7.99 \\
\hline
\end{tabular}

ature can be improved. As studied by Villiers, ${ }^{45}$ the original $\mathrm{SAFT}^{23,24}$ gives better isochoric heat capacity than PC-SAFT. Inclusion of isochoric heat capacity data into both universal constants regression and parameter estimation may be a useful approach for obtaining better parameters.

\section{CONCLUSION}

In this work, the performance of the SRK, CPA, and sPC-SAFT EoS is evaluated for speed of sound of normal alkanes and methanol. The results reveal that (i) none of the models could describe the speed of sound with satisfactory accuracy; (ii) fitting parameters to experimental data could improve the speed of sound for chain molecules, i.e. CPA performs better than SRK; (iii) sPC-SAFT is superior to SRK and CPA from both the accuracy and the curvature points of view over wide pressure ranges due to its theoretically more correct physical term. This indicates that the functional form of the model is more important than the parameter fitting strategy. So the sPCSAFT model was chosen as the starting point for developing an approach for speed of sound. First, the inclusion of speed of sound data into pure component parameters was tested. This approach could not improve the description of speed of sound for normal alkanes even at the cost of loss of accuracy for vapor pressure and liquid density. Second, a general approach was proposed to integrate the speed of sound data into both the universal constants regression and the pure component parameters estimation. The new universal constants and the corresponding pure component parameters significantly improve the speed of sound calculations while obtaining acceptable vapor pressure and liquid density. The new parameters exhibit good linear correlation functions for $m$, $m \sigma^{3}$, and $m \varepsilon / k$ with molecular weight. Although use of the new approach gave comparable isobaric heat capacity results as SAFT-VR Mie, they both perform less satisfactorily than original PC-SAFT for the investigated normal hydrocarbons. However, the accuracy of the isobaric heat capacity for the investigated components in this work is acceptable in many real applications. The similar isochoric heat capacity results with PC-SAFT using the new and old universal constants show that the parameters presented here have little impact on the temperature derivatives, which is consistent with the observations from Villiers ${ }^{45}$ that the original SAFT model gives better isochoric heat capacity description. The investigation of methanol shows that PC-SAFT could give satisfactory results when putting the speed of sound into the pure component parameter fitting, although using the new universal constants indeed improves the trend, especially at the high pressure regions. This fact reveals that better results could be obtained by putting the second derivative properties into parameter estimation if there are extra fitting parameters, i.e. association energy and volume here and the interaction width of the SAFTVR model in the important terms.

\section{ASSOCIATED CONTENT}

\section{Supporting Information}

$\% \mathrm{AAD}$ of vapor pressure, liquid density, and speed of sound comparison between SRK, CPA, and PC-SAFT for hydrocarbons (Table S1), the new universal constants (Table S2), the linear correlations of the three pure component parameters of hydrocarbons against molecular weight (Table S3), the \% $\mathrm{AAD}$ of the same properties comparison between different approaches for primary alcohols (Table S4), and the isobaric heat capacity comparison between original PC-SAFT, approach 2, and SAFT-VR Mie (Tables S5 and S6). This material is available free of charge via the Internet at http://pubs.acs.org.

\section{AUTHOR INFORMATION}

\section{Corresponding Author}

*Tel.: 045-45252859. Fax: 045-45882258. E-mail: gk@kt.dtu. $\mathrm{dk}$.

\section{Notes}

The authors declare no competing financial interest.

\section{ACKNOWLEDGMENTS}

The authors acknowledge Peter Jørgensen Herslund, Dr. Ioannis Tsivintzelis, and Prof. Michael L. Michelsen for many useful discussions and for providing some parameters. The Ph.D. project of Xiaodong Liang is funded by the Danish National Advanced Technology Foundation (DNATF) and the Department of Chemical and Biochemical Engineering, Technical University of Denmark, to whom the authors express their gratitude.

\section{LIST OF SYMBOLS}

$\mathrm{EoS}=$ equation $(\mathrm{s})$ of state

$\mathrm{CPA}=$ cubic plus association

SAFT $=$ statistical associating fluid theory

PC-SAFT $=$ perturbed-chain statistical associating fluid theory

sPC-SAFT $=$ simplified-perturbed-chain statistical associating fluid theory

$\mathrm{AAD}=$ average absolute deviation

$N=$ molecular numbers 
$R=$ gas constant

$T=$ temperature

$P=$ pressure

$V=$ total volume

$Z=$ compressibility factor

$u=$ speed of sound

$C_{V}=$ isochoric heat capacity

$C_{P}=$ isobaric heat capacity

$A^{\mathrm{r}}=$ residual Helmholtz free energy

$a^{\mathrm{r}}=$ molar residual Helmholtz free energy

$m=$ segment number

$x=$ molar fraction

$d=$ temperature-dependent segment diameter

$g_{i j}^{\text {hs }}=$ radial distribution function of hard sphere fluid

$I_{1}, I_{2}=$ dispersion terms in eqs 16 and 17

\section{Greek Letters}

$\rho=$ molar density

$\sigma=$ segment diameter ( $T$ independent)

$\varepsilon=$ segment energy

$\eta=$ packing fraction

\section{Superscripts}

hs $($ seg $)=$ hard sphere (segment) term of reduced residual Helmholtz free energy

hc = chain term of reduced residual Helmholtz free energy disp $=$ dispersion term of reduced residual Helmholtz free energy

assoc $=$ association term of reduced residual Helmholtz free energy

$A_{i}=$ association site index $A$ of component $i$

\section{REFERENCES}

(1) Meng, G. T.; Jaworski, A. J.; Dyakowski, T.; Hale, J. M.; White, N. M. Design and testing of a thick-film dual-modality sensor for composition measurements in heterogeneous mixtures. Meas. Sci. Technol. 2005, 16, 942.

(2) Meng, G. T.; Jaworski, A. J.; White, N. M. Composition measurements of crude oil and process water emulsions using thickfilm ultrasonic transducers. Chem. Eng. Process. 2006, 45, 383.

(3) Goodwin, A. R. H. Method and apparatus for measuring fluid density downhole. U.S. Patent 6,640,625, 2003.

(4) Machefer, S.; Schnitzlein, K. Inline concentration monitoring of binary liquid mixtures in the presence of a dispersed gas phase with a modified speed of sound immersion probe. Chem. Eng. Technol. 2007, 30, 1381.

(5) Durackova, S.; et al. Estimation of critical micellar concentration through ultrasonic velocity-measurements. J. Appl. Polym. Sci. 1995, 57, 639.

(6) Automatic Leak Detection Sonar, Sonardyne. http://www. sonardyne.com/ (accessed June 2012).

(7) Michelsen, M. L.; Mollerup, J. M. Thermodynamic Models: Fundamentals \& Computational Aspects; Tie-Line Technology: Holte, Denmark, 2007.

(8) Gregorowicz, J.; O’Conell, J. P.; Peters, C. J. Some characteristics of pure fluid properties that challenge equation-of-state models. Fluid Phase Equilib. 1996, 116, 94.

(9) Soave, G. Equilibrium constant from a modified Redlich-Kwong equation of state. Chem. Eng. Sci. 1972, 27, 1197.

(10) Peng, D. Y.; Robinson, D. B. A New Two-Constant Equaiton of State. Ind. Eng. Chem. Fund. 1976, 15, 59.

(11) Ye, S.; Lagourette, B.; Alliez, J.; Saint-Guirons, H.; Xans, P. Comparison with experimental data of ultrasound velocity in pure hydrocarbons calculated from equations of state. Fluid Phase Equilib. 1992, 74, 157.
(12) Faradonbeh, M. R.; Abede, J.; Harding, T. G. Comparative Study of Eight Cubic Equations of State for Predicting Thermodynamic Properties of Alkanes. Can. J. Chem. Eng., in press.

(13) Kontogeorgis, G. M.; Voutsas, E. C.; Yakoumis, I. V.; Tassios, D. P. An Equation of state for associating Fluids. Ind. Eng. Chem. Res. 1996, 35, 4310.

(14) Kontogeorgis, G. M.; Michelsen, M. L.; Folas, G. K.; Derawi, S.; von Solms, N.; Stenby, E. H. Ten Years with the CPA (Cubic-PlusAssociation) Equation of State. Part 1. Pure Compounds and SelfAssociating Systems. Ind. Eng. Chem. Res. 2006, 45, 4855.

(15) Kontogeorgis, G. M.; Michelsen, M. L.; Folas, G. K.; Derawi, S.; von Solms, N.; Stenby, E. H. Ten Years with the CPA (Cubic-PlusAssociation) Equation of State. Part 2. Cross-Associating and Multicomponent Systems. Ind. Eng. Chem. Res. 2006, 45, 4869.

(16) Gross, J.; Sadowski, G. Perturbed-Chain SAFT: An Equation of State Based on a Perturbation Theory for Chain Molecules. Ind. Eng. Chem. Res. 2001, 40, 1244.

(17) Gross, J.; Sadowski, G. Application of the Perturbed-Chain SAFT Equation of State to Associating Systems. Ind. Eng. Chem. Res. 2002, 41, 5510.

(18) von Solms, N.; Michelsen, M. L.; Kontogeorgis, G. M. Computational and Physical Perfomance of a Modified PC-SAFT Equation of State for Highly Asymmetric Systems and Associating Mixtures. Ind. Eng. Chem. Res. 2003, 42, 1098.

(19) Wertheim, M. S. Fluids with Highly Directional Attractive Forces. I. Statistical Thermodynamics. J. Stat. Phys. 1984, 35, 19.

(20) Wertheim, M. S. Fluids with Highly Directional Attractive Forces. II. Thermodynamic Perturbation Theory and Intergral Equations. J. Stat. Phys. 1984, 35, 35.

(21) Wertheim, M. S. Fluids with Highly Directional Attractive Forces. III. Multiple Attraction Sites. J. Stat. Phys. 1986, 42, 459.

(22) Wertheim, M. S. Fluids with Highly Directional Attractive Forces. IV. Equilibrium Polymerization. J. Stat. Phys. 1986, 42, 477.

(23) Chapman, W. G.; Jackson, G.; Gubbins, K. E. Phase Equilibira of Associating Fluids: Chain Molecules with Multiple Sites. Mol. Phys. 1988, 65, 1057.

(24) Jackson, G.; Chapman, W. G.; Gubbins, K. E. Phase Equilibria of Associating Fluids: Spherical Molecules with Multiple Bonding Sites. Mol. Phys. 1988, 65, 1.

(25) Chapman, W. G.; Gubbins, K. E.; Jackson, G.; Radosz, M. New Reference Equation of State for Associating Liquids. Ind. Eng. Chem. Res. 1990, 29, 1709.

(26) Huang, S. H.; Radosz, M. Equation of State for Smalll, Large, Polydisperse, and Associating Molecules. Ind. Eng. Chem. Res. 1990, 29, 2284.

(27) Huang, S. H.; Radosz, M. Equation of State for Small, Large Polydisperse, and Associating Molecules: Extension to Fluid Mixtures. Ind. Eng. Chem. Res. 1991, 30, 1994.

(28) Gil-Villegas, A.; Galinda, A.; Whitehead, P. J.; Mills, S. J.; Jackson, G.; Burgess, A. N. Statistical associating fluid theory for chain molecules with attractive potentials of variable range. J. Chem. Phys. 1997, 106, 4168.

(29) Galindo, A.; Davies, L. A.; Gil-Villegas, A.; Jackson, G. The thermodynamics of mixtures and the corresponding mixing rules in the SAFT-VR approach for potentials of variable range. Mol. Phys. 1998, 93, 241.

(30) Blas, F. J.; Vega, L. F. Thermodynamic Behaviour of Homonuclear and Heteronuclear Lennard-Jones Chains with Association Sites from Simulation and Theory. Mol. Phys. 1997, 92, 135.

(31) Blas, F. J.; Vega, L. F. Prediction of Binary and Ternary Diagrams Using the Statistical Associating Fluid Theory (SAFT) Equation of State. Ind. Eng. Chem. Res. 1998, 37, 660.

(32) Llovell, F.; Pamies, J. C.; Vega, L. F. Thermodynamic properties of Lennard-Jones chain molecules: Renormalization-group correlations to a modified statistical associating fluid theory. J. Chem. Phys. 2004, 121,10715

(33) Kontogeorgis, G. M; Folas, G. K. Thermodynamic models for industrial applications - from classical and advanced mixing rules to association theories; John Wiley and Sons: New York, 2010. 
(34) Lafitte, T.; Bessieres, D.; Pineiro, M. M.; Daridon, J. L. Simultaneous estimation of phase behaviour and second-derivative properties using statistical associating fluid theory with variable range approach. J. Chem. Phys. 2006, 124, 024509.

(35) Lafitte, T.; Pineiro, M. M.; Daridon, J. L.; Bessieres, D. A Comprehensive Description of Chemical Association Effects on Second Derivative Properties of Alcohols through a SAFT-VR approach. J. Phys. Chem. B. 2007, 111, 3447.

(36) Davies, L. A.; Gil-Villegas, A.; Jackson, G. Describing the properties of chains of segments interacting via soft-core potentials of variable range with the SAFT-VR approach. Int. J. Thermophys. 1998, 19,675 .

(37) Khammar, M.; Shaw, J. M. Speed of Sound Prediction in 1-nalcohol+n-alkane mixtures using a translated SAFT-VR-Mie equation of state. Fluid Phase Equilib. 2010, 288, 145.

(38) Llovell, F.; Vega, L. F. Prediction of Thermodynamic Derivative Properties of Pure Fluids through the Soft-SAFT Equation of State. J. Phys. Chem. B. 2006, 110, 11427.

(39) Llovell, F.; Peters, C. J.; Vega, L. F. Second-Order thermodynamic derivative properties of selected mixtures by softSAFT equation of state. Fluid Phase Equilib. 2006, 248, 115.

(40) Diamantonis, N. I.; Economou, I. G. Evaluation of Statistical Associating Fluid Theory (SAFT) and Perturbed Chain-SAFT Equations of State for the Calculation of Thermodynamic Derivative Properties of Fluids Related to Carbon Capture and Sequestration. Energy Fuels 2010, 25, 3334.

(41) Polishuk, I. Hybridizing SAFT and Cubic EOS: What Can Be Achieved? Ind. Eng. Chem. Res. 2011, 50, 4183.

(42) Polishuk, I. Semi-Theoretical Versus Entirely Empirical: Comparing SAFT + Cubic and Soave-Benedict-Webb-Rubin (SBWR) Equations of State. Ind. Eng. Chem. Res. 2011, 50, 11422.

(43) Polishuk, I. Till which pressures the fluid phase EOS models might stay reliable? J. Supercrit. Fluids. 2011, 58, 204.

(44) Polishuk, I.; Katz, M.; Levi, Y.; Lubarsky, H. Implementation of PC-SAFT and SAFT + Cubic for modeling thermodynamic properties of haloalkanes. I. 11 halomethanes. Fluid Phase Equilib. 2011, 316, 66.

(45) Villiers, A. J. de; Schwarz, C. E.; Burger A. J.; Kontogeorgis, G. $M$. Evaluation of the CPA, PC-SAFT and SAFT equations of state in predicting derivative properties of selected non-polar and hydrogenbonding compounds. Fluid Phase Equilib., submitted for publication.

(46) Villiers, A. J. de. Evaluation and improvement of the sPC-SAFT equation of state for complex mixtures. $\mathrm{PhD}$ Dissertation, Stellenbosch University, Stellenbosch, South Africa, 2011.

(47) Michelsen, M. L.; Hendriks, E. M. Physical properties from association models. Fluid Phase Equilib. 2001, 180, 165.

(48) Michelsen, M. L. Robust and Efficient Solution Procedures for Association Models. Ind. Eng. Chem. Res. 2006, 45, 8449.

(49) Lundstrøm, C.; Michelsen, M. L.; Kontogeorgis, G. M.; Pedersen, K. S.; Sørensen, H. Comparison of the SRK and CPA equations of state for physical properties of water and methanol. Fluid Phase Equilib. 2006, 247, 149.

(50) DIPPR 801 database, Design Institute for Physical Property Data. AIChE: New York, 1998.

(51) NIST Chemistry Webbook. http://webbook.nist.gov/chemistry (accessed June 2012)

(52) Badalyan, A. L.; Otpuschennikov, N. F. Sound Velocity and Some Thermodynamic Characteristics of Liquid Octane, Decane and Undecane at High Pressures. Izv. Akad. Nauk. SSSR, Fiz. Zemli. 1971, 6, 207.

(53) Khasanshin, T. S.; Shchamialiou, A. P.; Poddubskij, O. G. Thermodynamic Properties of Heavy n-Alkanes in Liquid State: nDodecane. Int. J. Thermophys. 2003, 24, 1277.

(54) Daridon, J. L.; Lagourette, B. Ultrasonic Velocity of Liquid Tridecane and Tetradecane as a function of Temperature and Pressure. High Temp. - High Press. 2000, 32, 83.

(55) Daridon, J. L.; Carrier, H.; Lagourette, B. Pressure Dependence of the Thermophysical Properties of n-Pentadecane and nHeptadecane. Int. J. Thermophys. 2002, 23, 697.
(56) Boelhouwer, J. W. M. Sound Velocities in and Adiabatic Compressibilities of Liquid Alkanes at Various Temperature and Pressures. Physica 1967, 34, 484.

(57) Dutour, S.; Daridon, J. L.; Lagourette, B. Pressure and Temperature Dependence of the Speed of Sound and Related Properties in Normal Octadecane and Nondecane. Int. J. Thermophys. 2000, 21, 173.

(58) Dutour, S.; Daridon, J. L.; Lagourette, B. Speed of sound, density, and compressibilities of liquid eicosane and docosane at various temperature and pressures. High Temp. - High Press. 2001, 33, 371.

(59) Ting, P. D.; Joyce, P. C.; Jog, P. K.; Chapman, W. G.; Thies, M. C. Phase equilibrium modeling of mixtures of long-chain and shortchain alkanes using Peng-Robinson and SAFT. Fluid Phase Equilib. 2003, 206, 267.

(60) Dutour, S.; Lagourette, B.; Daridon, J. L. High-pressure speed of sound and compressibilities in heavy normal hydrocarbons: $\mathrm{n}-\mathrm{C} 23 \mathrm{H} 48$ and n-C24H50. J. Chem. Thermodyn. 2001, 33, 765.

(61) Dutour, S.; Lagourette, B.; Daridon, J. L. High-pressure speed of sound, density and compressibilities in heavy normal paraffins: nC28H58 and n-C36H74. J. Chem. Thermodyn. 2002, 34, 475.

(62) More, J. J. Levenberg-Marquardt algorithm: implementation and theory; Technical Report, CONF-770636-1, Contract W-31-109-ENG38; Argonne National Lab., IL, 1977.

(63) Plantier, F.; Daridon, J. L.; Lagourette, B. Nonlinear parameter (B/A) measurements in methanol, 1-butanol and 1-octanol for different pressures and temperature. J. Phys. D: Appl. Phys. 2002, 35, 1063.

(64) Avlund, A. S. Extension of Association Models to Complex Chemicals. PhD Dissertation, Technical University of Denmark, Kgs. Lyngby, Denmark, 2011.

(65) Randzio, S. L.; Grolier, J. P. E.; Quint, J. R.; Eatough, D. J.; Lewis, E. A.; Hansen, L. D. n-Hexane as a Model for Compressed Simple Liquids. Int. J. Thermophys. 1994, 15, 415.

(66) Bessieres, D.; Saint-Guirons, H.; Daridon, J. L. High Pressure Measurement of $\mathrm{n}$-Dodecane Heat Capacity up to $100 \mathrm{MPa}$. Calculation from Equation of State. High Press. Res. 2000, 18, 279.

(67) Bessieres, D.; Saint-Guirons, H.; Daridon, J. L. Thermophysical Properties of n-Tridecane from 313.15 to $373.15 \mathrm{~K}$ and up to $100 \mathrm{MPa}$ from Heat Capacity and Density Data. J. Therm. Anal. Calorim. 2000, 62,621 . 